\title{
Current Status of Electromagnetic Particle Detectors for LHAASO-KM2A
}

\section{Jia Liu, ${ }^{a, b, *}$ Xiaopeng Zhang ${ }^{a, b}$ and Xiangdong Sheng ${ }^{a, b}$ on behalf of the LHAASO Collaboration}

(a complete list of authors can be found at the end of the proceedings)

${ }^{a}$ Key Laboratory of Particle Astrophyics, Institute of High Energy Physics, Chinese Academy of Sciences, 100049 Beijing, China.

${ }^{b}$ TIANFU Cosmic Ray Research Center, Chengdu, Sichuan, China.

E-mail: jialiu@ihep.ac.cn, zhangxp@ihep.ac.cn

The Large High Altitude Air Shower Observatory (LHAASO) is a new generation hybrid cosmic ray observatory which is expected to reveal the mystery of the origin of cosmic rays. The one square kilometer array (KM2A) containing 5195 Electromagnetic particle Detectors (ED) and 1188 Muon Detectors (MD) is a sub-array of the LHAASO. The EDs are designed to measure the density and arriving time of the secondary particles of cosmic rays.

KM2A reaches its 3/4 scale by December 2020. 3978 electromagnetic particle detectors (EDs) and 917 muon detectors (MDs) are now in stable operation. In this paper, we will introduce the construction process of ED, the performances and long-term stability of the detectors.

$37^{\text {th }}$ International Cosmic Ray Conference (ICRC 2021)

July 12th-23rd, 2021

Online - Berlin, Germany

\footnotetext{
*Presenter
} 


\section{Introduction}

The Large High Altitude Air Shower Observatory (LHAASO)[1], is a complex of extensive air shower (EAS) detector array, located at Mt. Haizi (29²1'27.6" N, 100 08'19.6" E) and the altitude of $4410 \mathrm{~m}$ above sea level (a.s.l.), in an area at the edge of the Qingzang plateau near Daocheng, Sichuan Province, China. It consists of an extensive air shower detector array covering an area of $1.3 \mathrm{~km}^{2}$ (KM2A) with 5195 scintillator counters (ED, $1 \mathrm{~m}^{2}$ active area) and 1188 muon detectors (MD, water Cherenkov detector with an area of $36 \mathrm{~m} 2$ buried under 2.5 meters of dirt). In the center of the array are the Water Cherenkov Detector Array (WCDA) covering 78,000 m2 and Wide Field-of-view air Cherenkov/fluorescence Telescope Array (WFCTA) of 18 telescopes. LHAASO is designed for detection of air showers induced by all kinds of cosmic-ray particles with energy ranging from a few tens of $\mathrm{GeV}$ to a few $\mathrm{EeV}$, and for very effectively identifying gamma rays out of the charged cosmic-ray background. LHAASO is, therefore, a multi-purpose facility for very-high-energy gamma ray astronomical observation, cosmic-ray energy spectrum measurements for individual species and other wide-ranging topics associated with the cosmic-rays or air-shower phenomena. For the gamma-ray source survey, the designed sensitivity is about $0.01 \mathrm{CU}$ (Crab Unit). Its wide FoV allows LHAASO to 67 survey $1 / 7$ of the northern sky at any moment for gammaray sources. With the operation in full duty cycle, LHAASO scans the entire northern hemisphere every 24 hours. The spectra of all sources in its FoV will be measured with high precision over a wide energy range from $10^{11} \mathrm{eV}$ to $10^{15} \mathrm{eV}$. These measurements, particularly above $100 \mathrm{TeV}$, will offer a great opportunity for finding cosmic-baryon PeVatrons and therefore identifying cosmic-ray origins among the gamma-ray sources. The Cherenkov/fluorescence telescopes of WFCTA are used to record the longitudinal development of the air showers that trigger the arrays on the ground. The combined detection of showers using all detectors in LHAASO enables the identification of the cosmic-ray species, at least for protons, $\mathrm{H}+\mathrm{He}$ and iron nuclei with an aperture greater than 4000 $\mathrm{m}^{2} \mathrm{sr}$. The knees of the spectra of cosmic rays are expected to be measured at energies above 100 $\mathrm{TeV}$ for individual mass groups.

\section{The electromagnetic particle detectors for LHAASO-KM2A}

KM2A, operating at an energy threshold of about $10 \mathrm{TeV}$, consists of 5195 electromagnetic particle detectors (ED) and 1188 underground muon detectors (MD)[2]. This array is devoted to the measurement of many air shower parameters, as energy, arrival direction and muon content, useful to characterize the primary particle inducing the shower. The ED is composed of four detection sensitive units, a photomultiplier tube (PMT) (XP3960[3], 9 linear focused dynode stages, 1.5-inch end window), an electronics system, a power supply system and the housing unit. The detection sensitive unit consists of a plastic scintillation tile $(100 \mathrm{~cm} \times 25 \mathrm{~cm} \times 1.0 \mathrm{~cm})$ wrapped with Tyvek and a layer of black cloth. Twelve $2.7 \mathrm{~m}$ long wavelength-shifting fibers are embedded in 24 grooves of the plastic scintillation tile. Each fiber passes in the tile twice through two grooves. All the ends of 96 fibers are bunched together to be polished and coupled directly to the PMT photocathode. Two output channels, the anode and the 6th dynode of the PMT, with different gains and linear response ranges are deployed to cover a large dynamic range. A layer of lead plate of $5 \mathrm{~mm}$ thickness is 
stacked on the plastic scintillation tile to convert gamma rays into electron-positron pairs to improve the angular resolution[4].

\section{Construction progress of ED array}

On September 30, 2019, the installation of 1/4 array had been completed and a total of 1320 detectors had been installed. The trigger event rate of the $1 / 4$ array is about $500 \mathrm{~Hz}$. From September 30, 2019 to December 15, 2019, a quarter of the array was put into trial operation, measuring about 3.5 billion cosmic ray events, generating 0.35T raw data every day. In December 2019 , the installation of $1 / 2$ array had been completed, with a total of 2373 detectors installed. The trigger event rate of the 1/2 array is about 900-1000hz. From December 27, 2019 to November 30, 2020 , the $1 / 2$ array measured about 26 billion cosmic ray events, generating $0.72 \mathrm{~TB}$ of raw data every day, with a total of about $260 \mathrm{~TB}$ of raw data. In early December 2020, the installation of $3 / 4$ array was completed, and 4008 ed units were installed. The trigger event rate of the 3/4 array reaches $1900 \mathrm{~Hz}$, generating about $1.45 \mathrm{~TB}$ of raw data per day. In July 1, 2021, the whole array had been completed and the ED array will be operated in the end of a month.

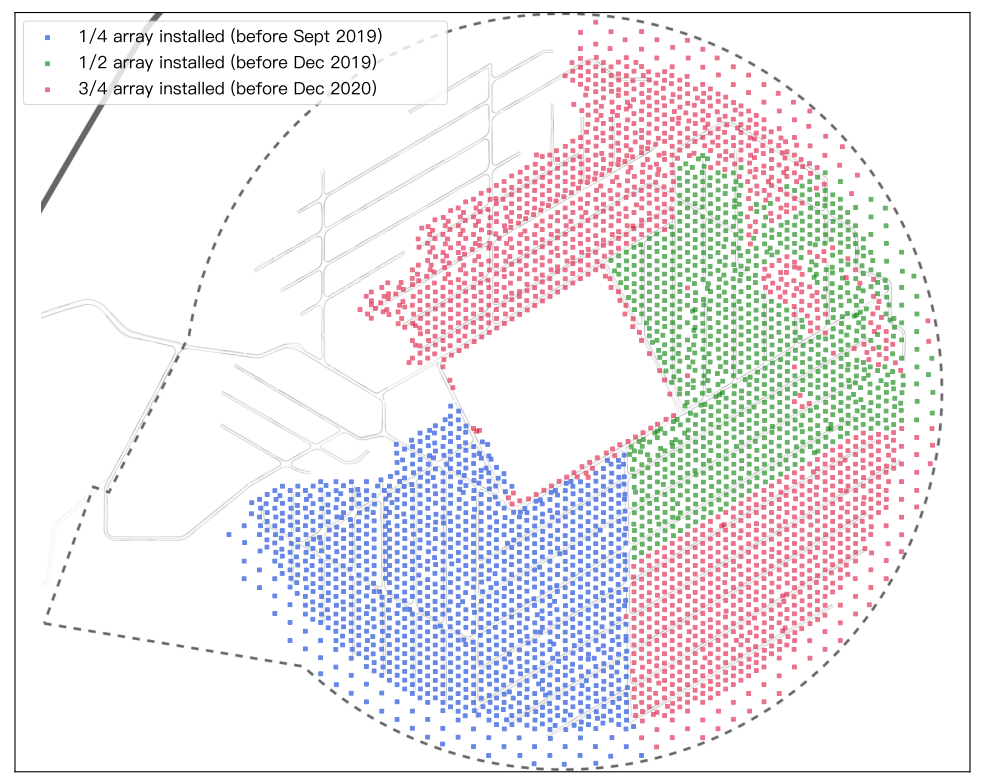

Figure 1: Construction process of the $3 / 4 \mathrm{ED}$ array

\section{Monitoring of the ED array}

To achieve a reliable and automatic procedure of monitoring on the large amount of data, a software package based on Python has been developed. It consists of two parts, online monitoring runs continuously on the DAQ server at the LHAASO site, and the offline monitoring analyzes the data and generates data reports on a daily basis. The main parameters in the monitoring and typical results are as follows. 


\subsection{Trigger rate}

Trigger rate or event rate is obtained by fitting the time interval distribution of triggered events (Figure 2). The typical value of event rate of 3/4 KM2A under the current configuration is $1.8 \mathrm{kHz}$ and fluctuates slightly over time by the influence of ambient temperature and atmospheric pressure (Figure 3).

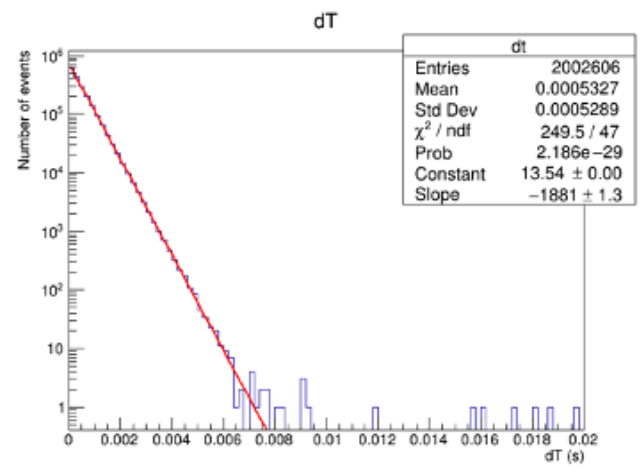

Figure 2: Time interval distribution of events

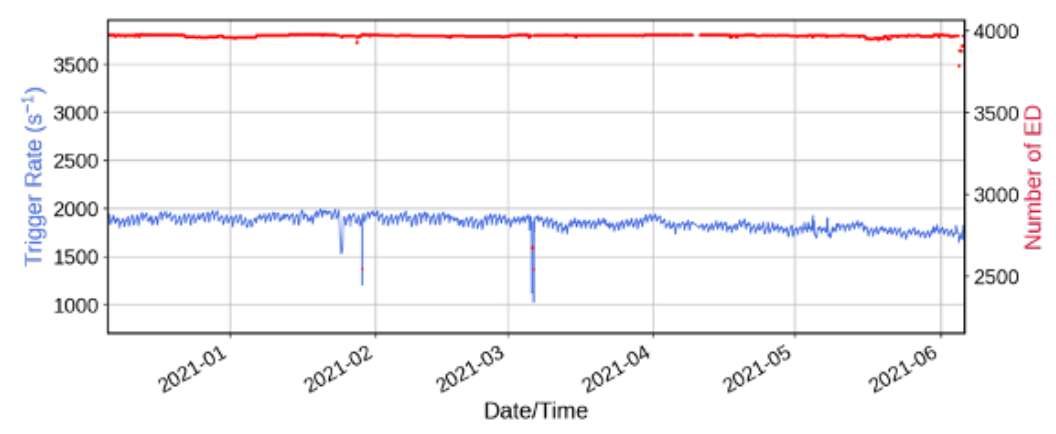

Figure 3: Trigger rate and number of EDs versus time

\subsection{Duty cycle}

The overall operation duty cycle of the array since June 2020 is $96.6 \%$. The daily duty cycle over date is shown in Figure 4. Routine maintenances, debugging of the DAQ system and accidental events such as power supply breakdown could reduce the operation duty cycle. Another minor cause to reduce the duty cycle is the automatic restart of a new DAQ run (usually lasts less than 10 $\min )$.

\subsection{Occupancy}

Occupancy is defined as the probability a specific detector is hit by particles in a triggered event. The occupancy of a detector depends on its geometry positions in the array. One typical spatial distribution of occupancies is shown in Figure 5. It can be seen that the peripheral EDs tend to have lower occupancies than EDs near the array center since they are less likely to participate in the trigger. A much higher or lower occupancy compared to surrounding EDs gives hint that the detector is in abnormal state, either having a high noise level or clock synchronization failure. 


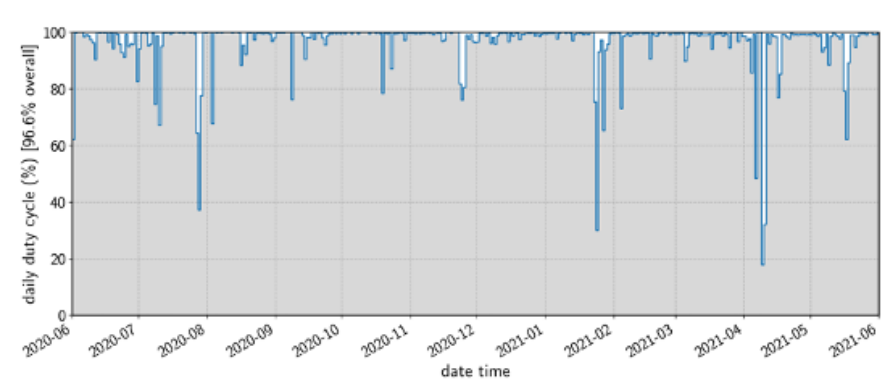

Figure 4: Operation duty cycle of 3/4 KM2A

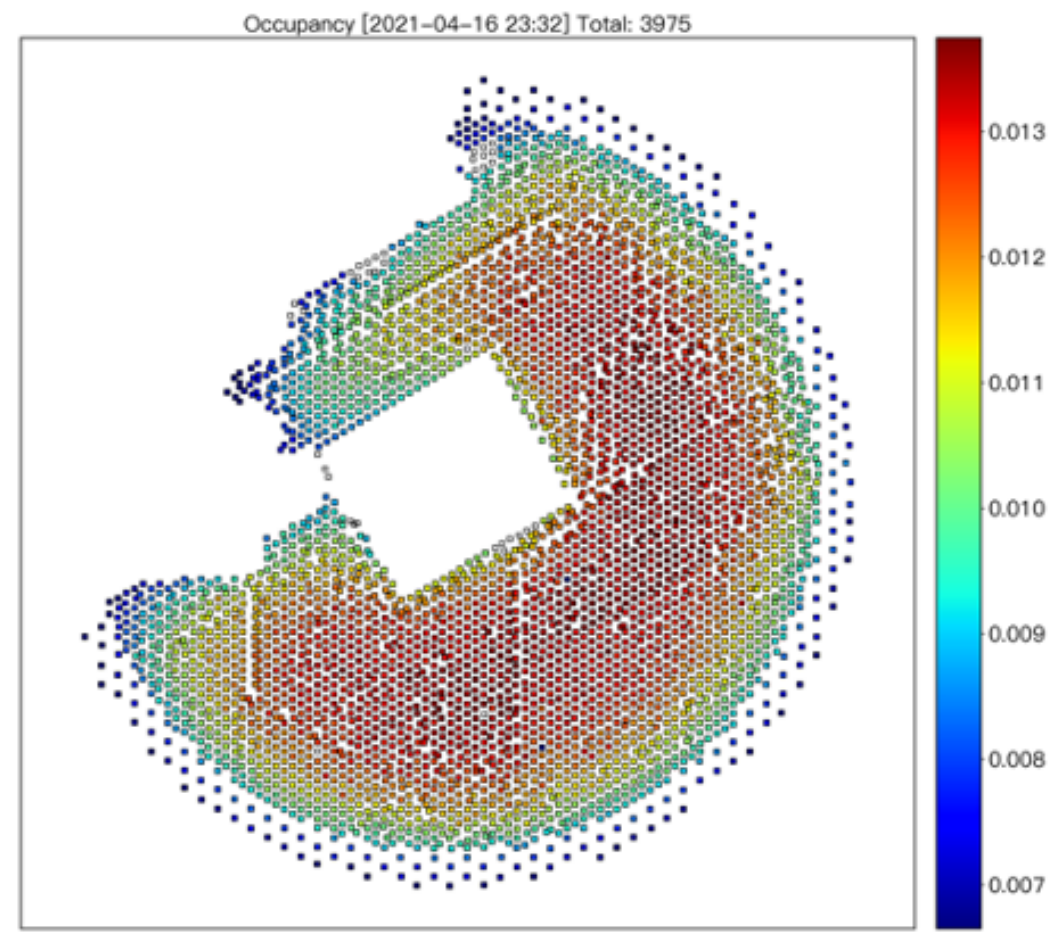

Figure 5: Spatial distribution of occupancies of EDs

\subsection{Charge MPV}

The most probable value of charge is obtained by fitting the single particle spectrum of one ED. The distribution of MPVs of all EDs is shown in Figure 6(a). For one of the EDs, the trend of the MPV over time is modulated by climate changes such as temperature and reflects its long-term stability (Figure 6(b)).

\section{Conclusion}

In the paper, the construction process of ED is presented. A software package based on Python has been developed to monitor the ED array. The performances of the detectors meet the deigned requirement and the detectors worked steadily. 


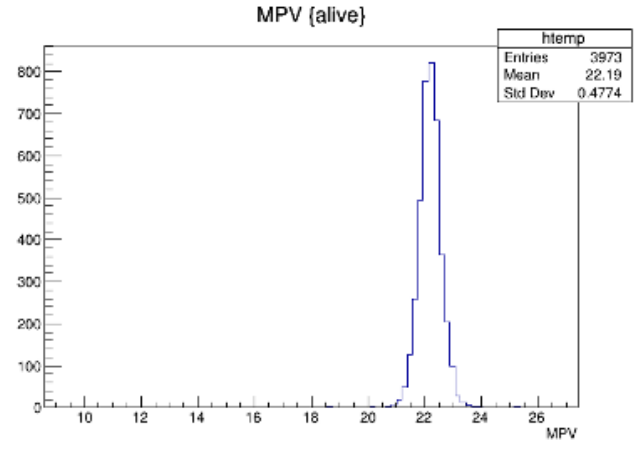

(a)

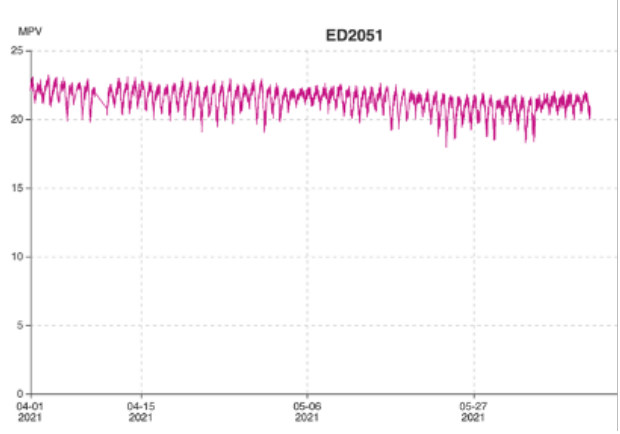

(b)

Figure 6: (a) Distribution of charge MPV of EDs (b) Long-term stability of MPV of one example ED

\section{Acknowledgments}

This work is supported by the Natural Sciences Foundation of China (No.11805209)

\section{References}

[1] Z. Cao, A future project at tibet: the large high altitude air shower observatory (LHAASO). Chinese Phys. C (2010) 34:249-252

[2] H. He, For the LHAASO Collaboration, Design of the LHAASO detectors, Radiation Detection Technology and Methods (2018) 2:7

[3] Y. Yu, H. Lv, D. Liu, X. Sheng, C. Feng, Characterization of the photomultiplier tube for the LHAASO electromagnetic particle detector, PoSICRC2019 (2019) 481

[4] J. Liu, A Study on the Effect of the Lead Plate on the Shower Front in the prototype array of LHAASO-KM2A, PoSICRC2015 (2016) 1007 


\section{Full Authors List: LHAASO Collaboration}

Zhen $\mathrm{Cao}^{1,2,3}$, F. Aharonian ${ }^{4,5}$, Q. An ${ }^{6,7}$, Axikegu $^{8}$, L.X. Bai ${ }^{9}$, Y.X. Bai ${ }^{1,3}$, L.X. Bai ${ }^{9}$, Y.X. Bai ${ }^{1,3}$, Y.W. Bao ${ }^{10}$, D. Bastieri ${ }^{11}$, X.J. Bi ${ }^{1,2,3}$, Y.J. Bi ${ }^{1,3}$, H. Cai ${ }^{12}$, J.T. Cai ${ }^{11}$, Zhe Cao ${ }^{6,7}$, J. Chang ${ }^{13}$, J.F. Chang ${ }^{1,3,6}$, B.M. Chen ${ }^{14}$, E.S. Chen ${ }^{1,2,3}$, J. Chen ${ }^{9}$, Liang Chen $^{1,2,3}$, Liang Chen ${ }^{15}$, Long $C^{1} n^{8}$, M.J. Chen ${ }^{1,3}$, M.L. Chen ${ }^{1,3,6}$, Q.H. Chen ${ }^{8}$, S.H. Chen ${ }^{1,2,3}$, S.Z. Chen ${ }^{1,3}$, T.L. Chen ${ }^{16}$,X.L. $\mathrm{Chen}^{1,2,3}$, Y. Chen $^{10}$, N. Cheng ${ }^{1,3}$, Y.D. Cheng ${ }^{1,3}$, S.W. Cui ${ }^{14}$, X.H. Cuii ${ }^{17}$, Y.D. Cui ${ }^{18}$, B. D'Ettorre Piazzoli1 ${ }^{19}$, B.Z. Dai ${ }^{20}$, H.L. Dai $^{1,3,6}$, Z.G. Dai ${ }^{7}$, Danzengluobu ${ }^{16}$, D. della Volpe ${ }^{21}$, X.J. Dong ${ }^{1,3}$, K.K. Duan ${ }^{13}$, J.H. Fan ${ }^{11}$, Y.Z. Fan ${ }^{13}$, Z.X. Fan ${ }^{1,3}$, J. Fang ${ }^{20}$, K. Fang $^{1,3}$, C.F. Feng ${ }^{22}$, L. Feng ${ }^{13}$, S.H. Feng ${ }^{1,3}$, Y.L. Feng ${ }^{13}$, B. Gao ${ }^{1,3}$, C.D. Gao ${ }^{22}$, L.Q. Gao ${ }^{1,2,3}$, Q. Gao ${ }^{16}$, W. Gao ${ }^{22}$, M.M. Ge $^{20}$, L.S. Geng ${ }^{1,3}$, G.H. Gong ${ }^{23}$, Q.B. Gou ${ }^{1,3}$, M.H. Gu ${ }^{1,3,6}$, F.L. Guo ${ }^{15}$, J.G. Guo ${ }^{1,2,3}$, X.L. Guo ${ }^{8}$, Y.Q. Guo ${ }^{1,3}$, Y.Y. Guo ${ }^{1,2,3,13}$, Y.A. $\mathrm{Han}^{24}$, H.H. He $\mathrm{H}^{1,2,3}$, H.N. He${ }^{13}$, J.C. He ${ }^{1,2,3}$, S.L. He ${ }^{11}$, X.B. He ${ }^{18}$, Y. He ${ }^{8}$, M. Heller ${ }^{21}$, Y.K. Hor ${ }^{18}$, C. Hou ${ }^{1,3}$, H.B. Hu ${ }^{1,2,3}$, S. $\mathrm{Hu}^{9}$, S.C. $\mathrm{Hu}^{1,2,3}$, X.J. Hu ${ }^{23}$, D.H. Huang ${ }^{8}$, Q.L. Huang ${ }^{1,3}$, W.H. Huang ${ }^{22}$, X.T. Huang ${ }^{22}$, X.Y. Huang ${ }^{13}$, Z.C. Huang ${ }^{8}$, F. Ji ${ }^{1,3}$, X.L. $\mathrm{Ji}^{1,3,6}$, H.Y. Jia ${ }^{8}$, K. Jiang ${ }^{6,7}$, Z.J. Jiang ${ }^{20}$, C. Jin ${ }^{1,2,3}$, T. Ke ${ }^{1,3}$, D. Kuleshov ${ }^{25}$, K. Levochkin ${ }^{25}$, B.B. Li ${ }^{14}$, Cheng Li ${ }^{6,7}$, Cong Li $^{1,3}$,

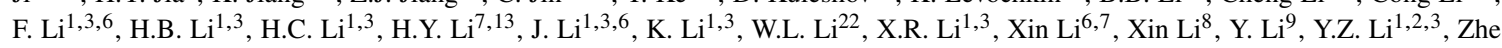

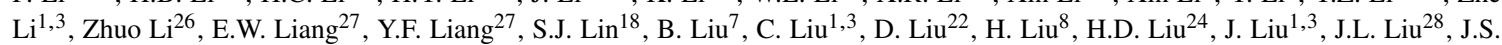

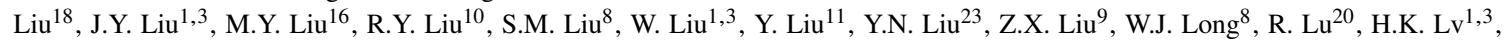

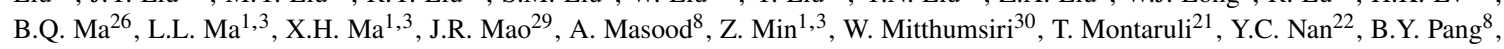
P. Pattarakijwanich ${ }^{30}$, Z.Y. Pei ${ }^{11}$, M.Y. Qi ${ }^{1,3}$, Y.Q. Qi ${ }^{14}$, B.Q. Qiao ${ }^{1,3}$, J.J. Qin ${ }^{7}$, D. Ruffolo ${ }^{30}$, V. Rulev ${ }^{25}$, A. Sáiz ${ }^{30}$, L. Shao ${ }^{14}$, O. Shchegolev $^{25,31}$, X.D. Sheng ${ }^{1,3}$, J.Y. Shi ${ }^{1,3}$, H.C. Song ${ }^{26}$, Yu.V. Stenkin ${ }^{25,31}$, V. Stepanov ${ }^{25}$, Y. Su ${ }^{32}$, Q.N. Sun ${ }^{8}$, X.N. Sun ${ }^{27}$, Z.B.

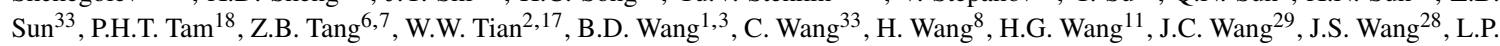
Wang $^{22}$, L.Y. Wang ${ }^{1,3}$, R.N. Wang ${ }^{8}$, W. Wang ${ }^{18}$, W. Wang ${ }^{12}$, X.G. Wang ${ }^{27}$, X.J. Wang ${ }^{1,3}$, X.Y. Wang ${ }^{10}$, Y. Wang ${ }^{8}$, Y.D. Wang ${ }^{1,3}$, Y.J. Wang $^{1,3}$, Y.P. Wang ${ }^{1,2,3}$, Z.H. Wang ${ }^{9}$, Z.X. Wang ${ }^{20}$, Zhen Wang ${ }^{28}$, Zheng Wang ${ }^{1,3,6}{ }^{3}$ D.M. Wei ${ }^{13}$, J.J. Wei ${ }^{13}$, Y.J. Wei ${ }^{1,2,3}$, T. Wen $^{20}$, C.Y. $\mathrm{Wu}^{1,3}$, H.R. Wu ${ }^{1,3}$, S. $\mathrm{Wu}^{1,3}$, W.X. Wu ${ }^{8}$, X.F. Wu ${ }^{13}$, S.Q. Xi ${ }^{1,3}$, J. Xia ${ }^{7,13}$, J.J. Xia ${ }^{8}$, G.M. Xiang ${ }^{2,15}$, D.X. Xiao ${ }^{16}$, G. Xiao ${ }^{1,3}$, H.B. Xiao ${ }^{11}$, G.G. Xin ${ }^{12}$, Y.L. Xin ${ }^{8}$, Y. Xing ${ }^{15}$, D.L. Xu ${ }^{28}$, R.X. Xu ${ }^{26}$, L. Xue ${ }^{22}$, D.H. Yann ${ }^{29}$, J.Z. Yan ${ }^{13}$, C.W. Yang 9 , F.F. Yang ${ }^{1,3,6}$, J.Y. Yang ${ }^{18}$, L.L. Yang ${ }^{18}$, M.J. Yang ${ }^{1,3}$, R.Z. Yang ${ }^{7}$, S.B. Yang ${ }^{20}$, Y.H. Yao ${ }^{9}$, Z.G. Yao ${ }^{1,3}$, Y.M. Ye ${ }^{23}$, L.Q. Yin ${ }^{1,3}$, N. Yin ${ }^{22}$, X.H. You $^{1,3}$, Z.Y. You ${ }^{1,2,3}$, Y.H. Yu ${ }^{22}$, Q. Yuan ${ }^{13}$, H.D. Zeng ${ }^{13}$, T.X. Zeng ${ }^{1,3,6}$, W. Zeng ${ }^{20}$, Z.K. Zeng ${ }^{1,2,3}$, M. Zha ${ }^{1,3}$, X.X. Zhai ${ }^{1,3}$, B.B. Zhang $^{10}$, H.M. Zhang ${ }^{10}$, H.Y. Zhang ${ }^{22}$, J.L. Zhang ${ }^{17}$, J.W. Zhang ${ }^{9}$, L.X. Zhang ${ }^{11}$, Li Zhang ${ }^{20}$, Lu Zhang ${ }^{14}$, P.F. Zhang ${ }^{20}$, P.P. Zhang ${ }^{14}$, R. Zhang ${ }^{7,13}$, S.R. Zhang ${ }^{14}$, S.S. Zhang ${ }^{1,3}$, X. Zhang ${ }^{10}$, X.P. Zhang ${ }^{1,3}$, Y.F. Zhang ${ }^{8}$, Y.L. Zhang ${ }^{1,3}$, Yi Zhang ${ }^{1,13}$, Yong Zhang ${ }^{1,3}$, B. Zhao $^{8}$, J. Zhao ${ }^{1,3}$, L. Zhao ${ }^{6,7}$, L.Z. Zhao ${ }^{14}$, S.P. Zhao ${ }^{13,22}$, F. Zheng ${ }^{33}$, Y. Zheng ${ }^{8}$, B. Zhou ${ }^{1,3}$, H. Zhou ${ }^{28}$, J.N. Zhou ${ }^{15}$, P. Zhou ${ }^{10}$, R. Zhou $^{9}$, X.X. Zhou ${ }^{8}$, C.G. Zhu ${ }^{22}$, F.R. Zhu ${ }^{8}$, H. Zhu ${ }^{17}$, K.J. Zhu ${ }^{1,2,3,6}$ and X. Zuo ${ }^{1,3}$

${ }^{1}$ Key Laboratory of Particle Astrophyics \& Experimental Physics Division \& Computing Center, Institute of High Energy Physics, Chinese Academy of Sciences, 100049 Beijing, China. ${ }^{2}$ University of Chinese Academy of Sciences, 100049 Beijing, China. ${ }^{3}$ TIANFU Cosmic Ray Research Center, Chengdu, Sichuan, China. ${ }^{4}$ Dublin Institute for Advanced Studies, 31 Fitzwilliam Place, 2 Dublin, Ireland. ${ }^{5}$ Max-Planck-Institut for Nuclear Physics, P.O. Box 103980, 69029 Heidelberg, Germany. ${ }^{6}$ State Key Laboratory of Particle Detection and Electronics, China. ${ }^{7}$ University of Science and Technology of China, 230026 Hefei, Anhui, China. ${ }^{8}$ School of Physical Science and Technology \& School of Information Science and Technology, Southwest Jiaotong University, 610031 Chengdu, Sichuan, China. ${ }^{9}$ College of Physics, Sichuan University, 610065 Chengdu, Sichuan, China. ${ }^{10}$ School of Astronomy and Space Science, Nanjing University, 210023 Nanjing, Jiangsu, China. ${ }^{11}$ Center for Astrophysics, Guangzhou University, 510006 Guangzhou, Guangdong, China. ${ }^{12}$ School of Physics and Technology, Wuhan University, 430072 Wuhan, Hubei, China. ${ }^{13}$ Key Laboratory of Dark Matter and Space Astronomy, Purple Mountain Observatory, Chinese Academy of Sciences, 210023 Nanjing, Jiangsu, China. ${ }^{14}$ Hebei Normal University, 050024 Shijiazhuang, Hebei, China. ${ }^{15}$ Key Laboratory for Research in Galaxies and Cosmology, Shanghai Astronomical Observatory, Chinese Academy of Sciences, 200030 Shanghai, China. ${ }^{16}$ Key Laboratory of Cosmic Rays (Tibet University), Ministry of Education, 850000 Lhasa, Tibet, China. ${ }^{17}$ National Astronomical Observatories, Chinese Academy of Sciences, 100101 Beijing, China. ${ }^{18}$ School of Physics and Astronomy \& School of Physics (Guangzhou), Sun Yat-sen University, 519000 Zhuhai, Guangdong, China. ${ }^{19}$ Dipartimento di Fisica dell'Università di Napoli ‘Federico II”, Complesso Universitario di Monte Sant’Angelo, via Cinthia, 80126 Napoli, Italy. ${ }^{20}$ School of Physics and Astronomy, Yunnan University, 650091 Kunming, Yunnan, China. ${ }^{21}$ D'epartement de Physique Nucl'eaire et Corpusculaire, Facult'e de Sciences, Universit'e de Gen'eve, 24 Quai Ernest Ansermet, 1211 Geneva, Switzerland. ${ }^{22}$ Institute of Frontier and Interdisciplinary Science, Shandong University, 266237 Qingdao, Shandong, China. ${ }^{23}$ Department of Engineering Physics, Tsinghua University, 100084 Beijing, China. ${ }^{24}$ School of Physics and Microelectronics, Zhengzhou University, 450001 Zhengzhou, Henan, China. ${ }^{25}$ Institute for Nuclear Research of Russian Academy of Sciences, 117312 Moscow, Russia. ${ }^{26}$ School of Physics, Peking University, 100871 Beijing, China. ${ }^{27}$ School of Physical Science and Technology, Guangxi University, 530004 Nanning, Guangxi, China. ${ }^{28}$ Tsung-Dao Lee Institute \& School of Physics and Astronomy, Shanghai Jiao Tong University, 200240 Shanghai, China. ${ }^{29}$ Yunnan Observatories, Chinese Academy of Sciences, 650216 Kunming, Yunnan, China. ${ }^{30}$ Department of Physics, Faculty of Science, Mahidol University, 10400 Bangkok, Thailand. ${ }^{31}$ Moscow Institute of Physics and Technology, 141700 Moscow, Russia. ${ }^{32}$ Key Laboratory of Radio Astronomy, Purple Mountain Observatory, Chinese Academy of Sciences, 210023 Nanjing, Jiangsu, China. ${ }^{33}$ National Space Science Center, Chinese Academy of Sciences, 100190 Beijing, China. 